\title{
Prague Loretto - Measurements of Moisture Content in Sculptural Group Material
}

\section{J. Římal}

An innovative method of spatial measurement of the moisture distribution in a sculptural group has been developed and verified. This method facilitates monitoring of moisture distribution and, therefore, the condition of the sculptural group in a spatial perspective.

The seven-month measurements were in order to take into account all climatic effects influencing the sculpture.

These measurements resulted from the practical requirement to provide source material for future conservation interventions to be conducted on the sculptural group in the Prague Loretto.

Keywords: Prague Loretto, measurement of moisture fields, mass transport.

\section{Introduction}

The requirement to measure the material moisture content of a sculptural group within the Prague Loretto complex was based on its condition in 1991. The sculptural group was in critical condition with typical symptoms of deep sandstone degradation in the form of chinks, cracks and surface spalling of the larger parts.

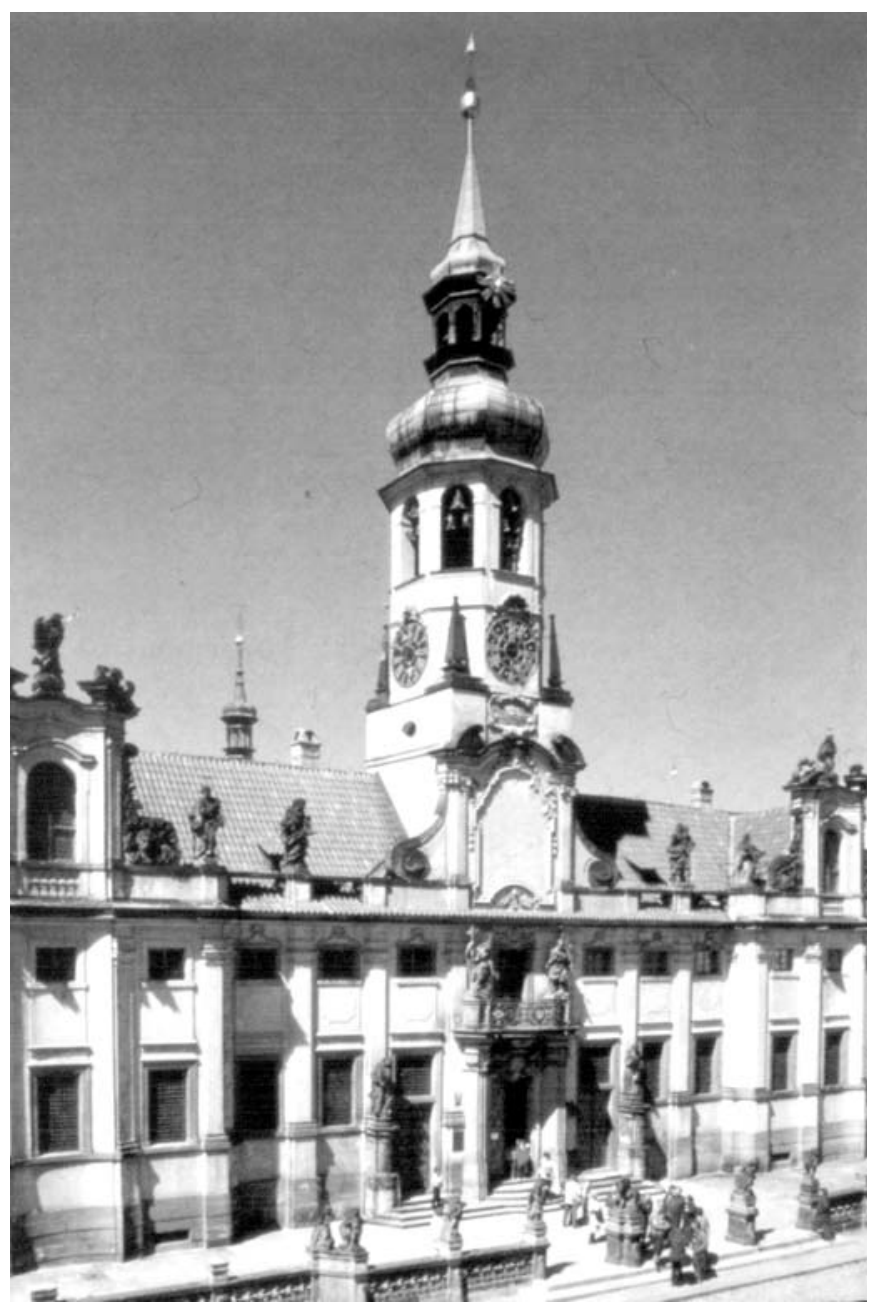

Fig. 1: Prague Loretto

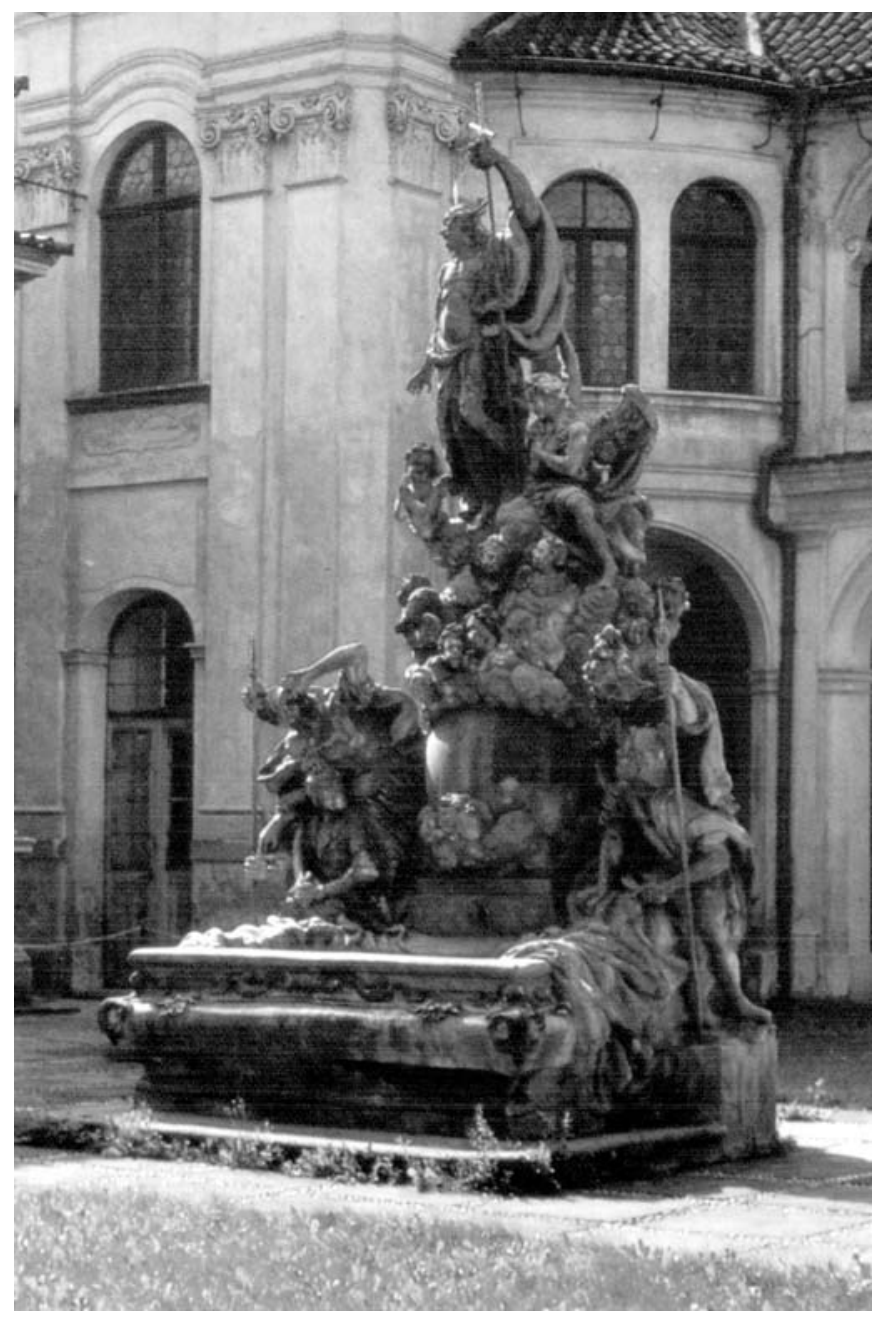

Fig. 2: Foutain decorated with the sculpture of the Resurrection of Our Lord - Jan Michael Brüderle and Richard Prachner, (1739-1740)

The objective causes of this condition were directly connected with the properties of the sandstone, which is relatively heterogeneous, with numerous hydrolytic transformations of ferrous minerals (limonite). Erosive failures had presumably also occurred in the sandstone in the more distant past, always provoked by water, and several attempts had already been made to conserve the sculptural group. 
Previous conservation attempts on the sculptural group are the second, subjective cause of its contemporary dilapidated condition. The remains of conservation attempts can still be identified, namely in the form of fragments of various fillings and surface crusts, originally meant in good faith, but which today are the fundamental sources of secondary erosion and corrosion processes.

These interventions, in particular, have violated the basic requirements for natural water migration within the porous structure of the stone. In turn, water tends to accumulate in various intermediate stages of the natural stone, and in its artificial forms. This has produced the usual negative effects, such as frost heaving pressures during winter periods, hydrolytic effects, hydration and crystallization pressures, etc.

It is obvious that the condition is not improved by the increased moisture content in the stone, due to both storm water and capillary elevation from the subsoil of the sculptural group. There is nowadays heavy air pollution in Prague, and special in the Hradčany locality. All types of water within the stone porous system in addition to the above-mentioned negative effects, develop into sorption or absorption centres for the cumulation of acid emissions. It is therefore necessary to know the exact values of the mass moisture content and, on the basis of the knowledge, to design measures for long-term protection of the sculptural group.

Based on this critical assessment of the situation and additional observations of the sculptural group, an extensive programme was designed in agreement with the monastery management, with the aim of creating a descriptive model of the condition of the entire volume of the sculptural group with regard to distribution of moisture maps inside the structure in relation to climatic conditions, namely, rainfall intensity and moisture content due to capillary elevation from the subsoil.

\section{Measurement technique}

In was decided to select a conductometric method for the measurements. This method involves low input voltage and the use of copper electrodes fixed at pre-selected points of the sculptural group that remained stable through out the year. In the given configuration, the method is highly accurate (the slight changes in polarization and corrosion potentials of the copper electrodes in the course of the year may be neglected), allowing, in particular, an investigation of the entire structure. By combining the measured points (electrodes) into electrode pairs and making sensitive measurement of the current flowing between them, practically all vertical and horizontal cross sections of the sculptural group could be mapped.

The probes consisted of copper wire with a diameter of $2 \mathrm{~mm}$ and a length of $60 \mathrm{~mm}$ mounted by means of lime dab into previously prepared boreholes with a diameter of $3 \mathrm{~mm}$. This type of copper pin anchorage guarantees an alkaline environment within the immediate vicinity of the electrodes for a period of at least one year, thus stabilizing the electrolytic medium in terms of appearance of variable polarization and corrosion potentials. The points for anchoring the boreholes and electrodes were selected with due care in order not to damage the original, valuable relief surface and also to include the points exposed to rainfall or, by contrast, those situated in rainfall shades, within all horizontal and vertical sections of the sculptural group. The measurements of the currents were implemented by a digital system using apparatus by SYSTEM ULTRAKUST (FRG), MULTIMETER (UK) and SANWA ELECTRIC (Japan) with an input working potential of $2 \mathrm{~V}$, which, at currents ranging up to $10 \mathrm{~mA}$, ensured only slight excess values of generated hydrogen voltage.

The use of copper electrodes of the first type was preferred to electrodes of the second type with electrolytic bridges. The latter type of electrodes have the fundamental advantage of eliminating polarization voltages, but, over a longer period of time, they show changes in electrolyte concentrations and potentially also in electrolyte volumes, which may subsequently have a decisive effect on the values of the read currents. Copper electrodes may be regarded as the most suitable solution as, when used in a sandstone medium with the respective moisture contents, they provide defined coverage with corrosion films, which, secondarily, render the surface passive and thus provide long-term electric stabilization.

The used configuration even allowed us to measure the surface layer of the sculptural group by the neighbouring electrodes, which was of interest namely during the period immediately following rainfall.

The distribution of the selected measured points is indicated in Fig. 2. In all, 14 measured points were numbered from the bottom edge of the sculptural group almost to the top of it.

Moisture content gauging was performed using the samples of original sandstone after it has been bored off. The samples came both from the socle and from the scaled layers. Simple analyses did not show extreme contents of free anions, which could, namely in heterogeneous distribution within the volume of the sculptural group, considerably affect the conductometric data. Gravimetric gauging using equally fixed electrodes in the original sandstone samples practically eliminated all negative effects of electrolytic interfaces. In fact, it turned out during the measurements that only about two electrode combinations showed some signs of fluctuations in the results, namely after heavy moistening, which could be attributed to electrolytic concentration effects.

\section{Results of measurements}

The results of the measurements are summed up in a graphic form (see Graph No. 1, No. 2). The charts treat the actual values of the mass moisture content in various ways in relation to the time of measurement and the mutual positions of the measured points, over a seven-month period in order to show a nearly cycle, including spring, summer and autumn. Graph 2 shows the gradients of the moisture field measured at a certain selected point in relation to other (14) points measured all over the statue.

The charts make it clear that we managed to collect complete sets of moisture data for the measured period. The analysis and interpretation enables us to draw wider conclusions on the moisture distribution inside the sculptural group and on the movement of the moisture zones inside the structure during one season of the year. It is also evident how the sculptural group reacted to heavy rainfall and how the water 


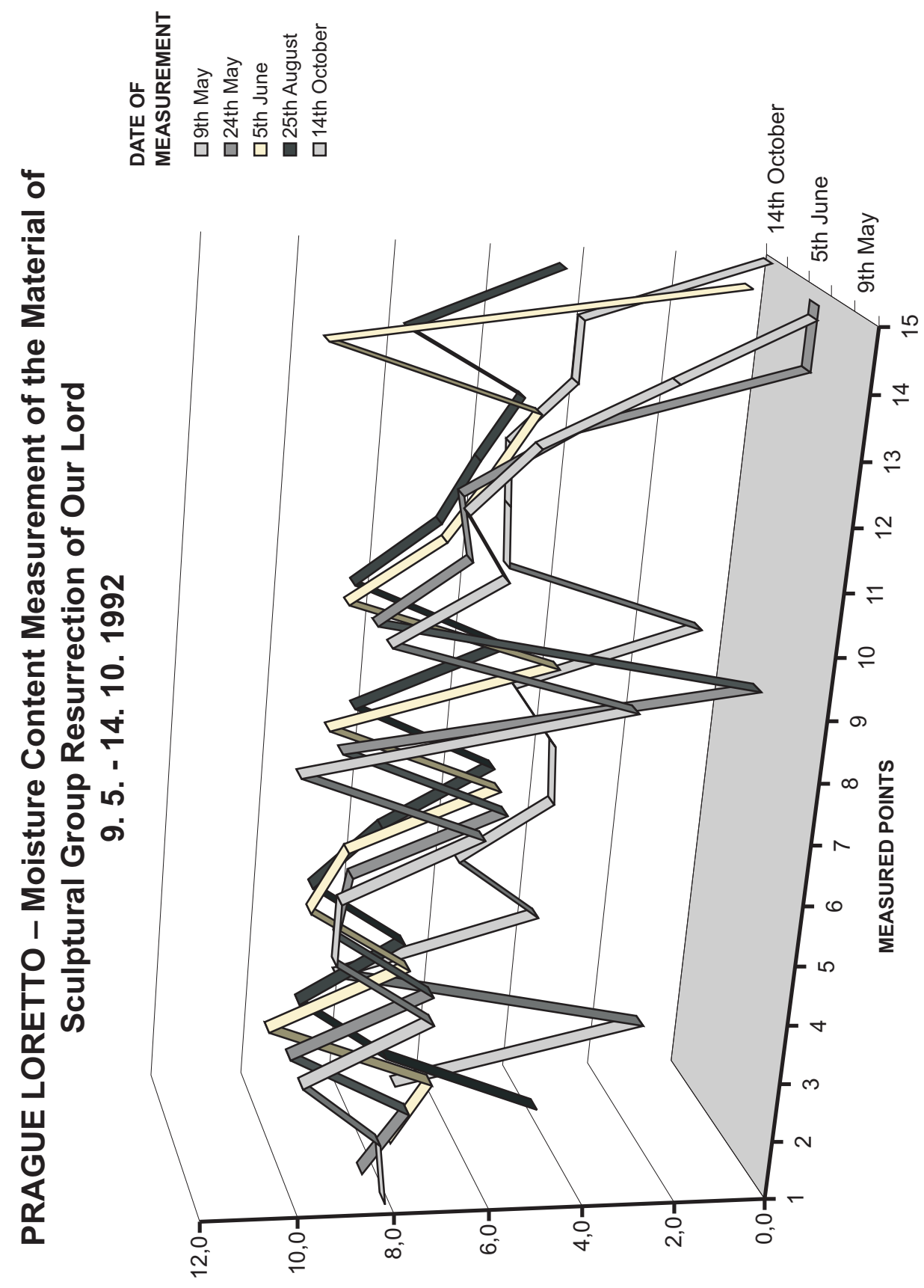

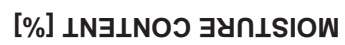




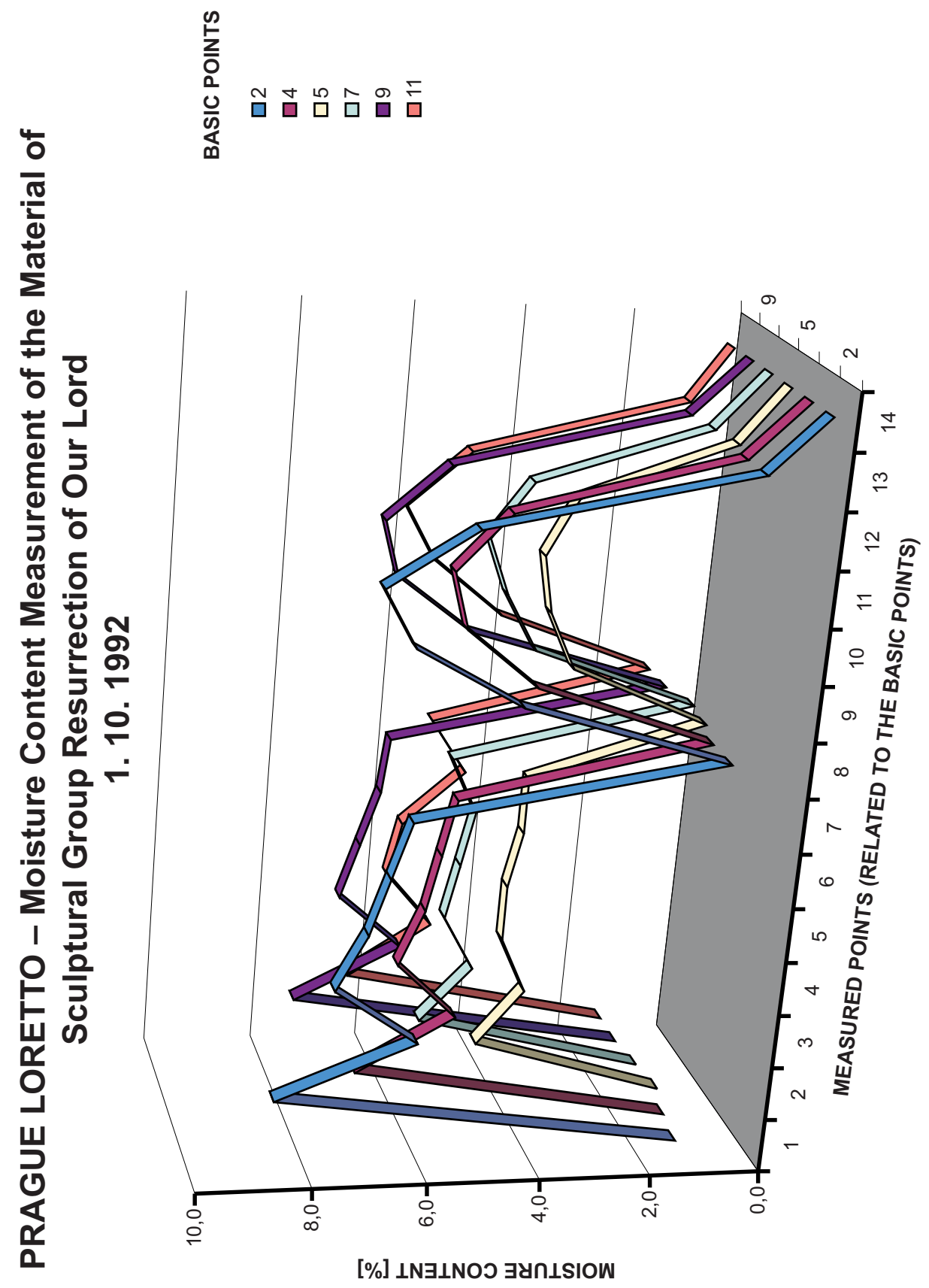


moved or was retained inside chinks and caverns, or how it migrated through the porous sandstone system. A study of the moisture profiles enabled the water contributions due to capillary elevation and rainfall to be estimated.

Though some combinations of measured points show considerable fluctuations of values depending on the actual moisture contents, it is possible to trace the basic moistening trends during the different seasons and also during the period of intensive summer drying up of the surface parts and their successive wetting after a heavy downpour.

In absolute values, the highest mass contents range above $10 \%$, but with regard to the erosion and corrosion behaviour of the sandstone, the steady average moistening of roughly the lower half of the sculptural group ranging from 6 to $8 \%$ seems to be more dangerous, as this is roughly twice the value of the corresponding balanced moisture content of the respective sandstone. These values of moistening are probably the basic source of failures in the intermediate material stages of the structure.

\section{Conclusion}

The above-described measurements of the mass moisture content values in the sculptural group Resurrection of Our Lord, located in the Paradise court of the Prague Loretto show that the structure is exposed to excessive moistening practically throughout the seven-month cycle. On average, the values of sandstone moistening are twice higher than would correspond to its average balanced contents.

The basic source of the current moistening of the lower parts of the sculptural group is capillary elevation of water from the subsoil. Contributions after rainfall cause high-level sandstone saturation, reaching up to values of around $12 \%$ of the mass moisture content, which must be regarded as critical moistening, namely in connection with the existence of watertight fillings and surface crusts.

It should be emphasised that it is water in its various forms within the porous sandstone structure that is causing accelerating damage to the sculptural group.

To conclude, we recommend that long-term rehabilitation measures on the sculptural group require the installa- tion of a damp-proofing barrier against moisture capillary elevation from the subsoil, and at the same time, the installation of roofing above the structure. Only complete elimination of excessive moisture sources can guarantee the success of any preservation or conservation interventions that will be needed on the sculptural group.

\section{Acknowledgement}

This project is being carried out with the participation of Ph.D. students Ing. Jan Pytel and Ing. Jana Zaoralová and undergraduate student Jakub Římal.

This research has been supported by the Grant Agency of the Czech Republic under grant No. 103/00/0776

\section{References}

[1] Groot, de S. R., Mazur, J.: Non - equilibrium thermodynamics. Amsterodam: North-Holand Publ. Co., 1962.

[2] Ǩímal, J.: Mass and Heat Transport in Building and Bridge Structures. Proceedings of Workshop 2000, Vol. B., Prague: CTU, 2000.

[3] Ǩímal, J.: Research of Impact of Temperature and Moisture Fields on the Bridge Structure of Charles Bridge. New Requirements for Materials and Structures, Prague: CTU, 1998, p. 105-110, ISBN 80-01-01838-5.

[4] Šejnoha, J., Bittnar, Z.: Numerical Methods in Structural Analysis. New York: ASCE Press, / London: Thomas Telford, 1996.

[5] Vodák, F.: Termodynamika spojitého prostředí. Prague: CTU, 1992.

Doc. RNDr. Jaroslav Římal, DrSc.

phone: +420224354702

fax: +420233333226

e-mail: rimal@fsv.cvut.cz

Czech Technical University

Faculty of Civil Engineering

Thákurova 7

166 29, Prague 6, Czech Republic 